\title{
Antibacterial Property of Essential Oil and its vibrational analysis
}

\author{
${ }^{1}$ S. Dheivamalar, ${ }^{2}$ V. Silambarasan, ${ }^{3}$ M.Gandhimathi, ${ }^{4}$ K. Vijayalakshmi \\ ${ }^{1}$ Department Of Physics Govt. Arts College for Women,Pudukkottai 62200,.India. \\ ${ }^{2}$ Department Of Physics, Sathyabama University, Chennai 600119,India \\ 3Crystal Growth Center, Anna University, Chennai 600025, India \\ ${ }^{4}$ Department Of Physics, Bishop Heber collge, Tiruchirappalli 620017,India.
}

\begin{abstract}
In recent years there has been an increasing interest in the use of natural substances, and some questions concerning the safety of synthetic compounds have encouraged more detailed studies of plant resources. Essential oils, odorous and volatile products of plant secondary metabolism, have a wide application in folk medicine, food flavoring and preservation as well as in fragrance industries. In our present work with the vibrational spectroscopy of quantum chemical calculations of Eugenol, Geronial have been carried out. The compounds are responsible for most of the characteristic aroma.DFT analysis is performed before and after application of clove oil on Asbergillus fungi..Vibrational analysis on Eugenol( 4-allyl-2-methoxyphenol is also carried out.The fluorescent property of the material was also discussed.
\end{abstract}

Key words: Essential oils, Antibacterial activity, Antifungal activity ,Eugenol (4-allyl-2-methoxyphenol)

\section{Introduction:}

The antimicrobial properties of essential oils have been known for many centuries. In recent years (1987-2001) a large number of essential oils and their constituents have been investigated for their antimicrobial properties against some bacteria and fungi in more than 500 reports.(1-5). This paper gives an overview on the susceptibility of human and food-borne bacteria and fungi towards different essential oils and their constituents.(6-8). This work deals with the vibrational spectroscopy of Eugenol by means of quantum chemical calculations. The mid and far FTIR and FT-Raman spectra were measured in the condensed state. The compound Eugenol (4-allyl-2-methoxyphenol) is responsible for most of the characteristic aroma of cloves.DFT analysis is performed before and after application of clove oil on Asbergillus fungi.Vibrational analysis on 4allyl-2-methoxyphenol is also carried out.The fluorescent property of the material was also discussed. Clove oil contained its principal constituent of Eugenol .

The clove oil were obtained from Lancaster Chemical Company, UK and used as such for the spectral measurements. The room temperature Fourier transform IR spectra of title compounds were measured in the $4000-50 \mathrm{~cm}^{-1}$ region at a resolution of $\pm 1 \mathrm{~cm}^{-1}$ using BRUKER IFS 66V Fourier transform spectrometer equipped with an MCT detector a $\mathrm{KBr}$ beam splitter and globar source. The Far IR spectrum was recorded on the same instrument using polyethylene pellet technique.

The Optimised energy structure is given in the Fig.1.

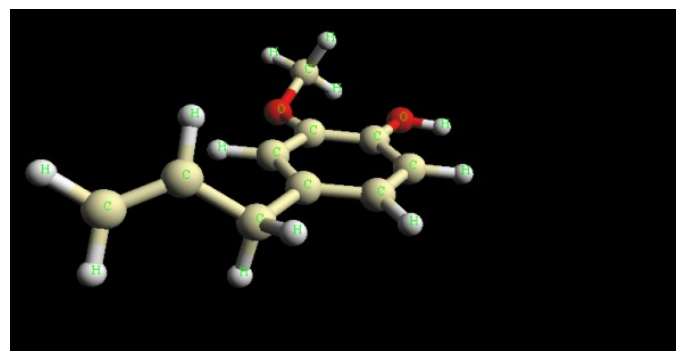

Fig.1.Optimisation of clove oil.

The Fig.2 shows the structure of Aspergillus fungai in bread.The experiment is done on the fungi by applying clove oil has its major constituents such as Eugenol.The FTIR study is also carried out to show the effect of clove oil before and after applying clove oil. 


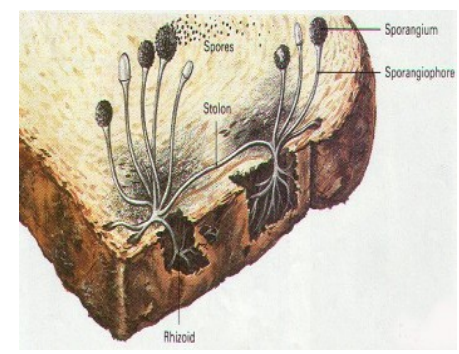

Fig.2.growth of Aspergillus fungai in bread

The florescent property of the clove oil also was carried to show the electron transition during the reaction clove oil on Aspergillus fungi.The Homo energy,Lumo energy of the molecule was also carried out to show the property of electron transfer.

\section{Electron transformation in HOMO state}

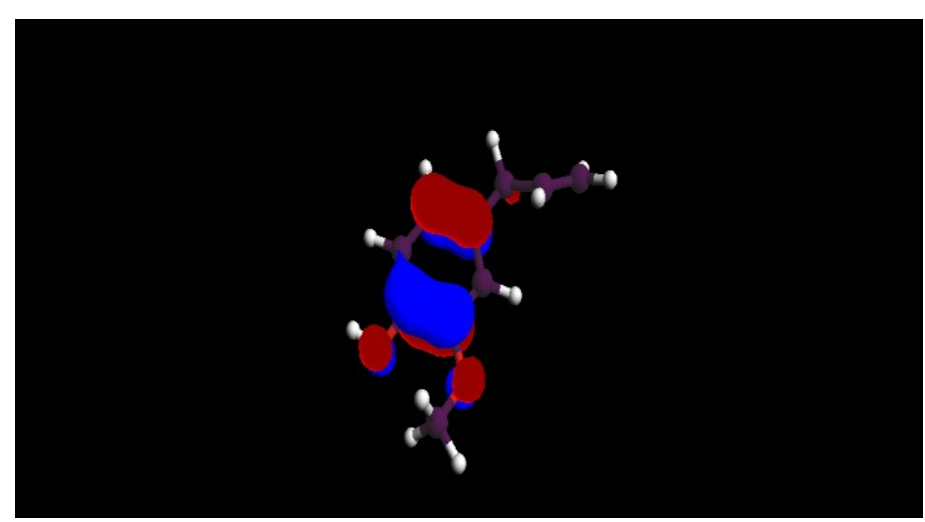

Electron spin polarization of clove oil

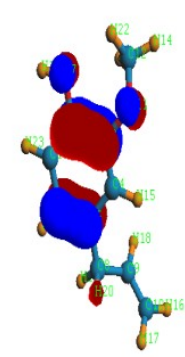

Vibrational analysis OF CLOVE OIL using FTIR METHOD:

\begin{tabular}{|l|l|l|l|}
\hline \multicolumn{2}{|l|}{ Vibratial assignments in clove oil } & \multicolumn{2}{l|}{$\begin{array}{l}\text { Vibrational analysis of Aspergillus fungi in } \\
\text { the presence of clove oil }\end{array}$} \\
\hline Frequency in cm & Assignment of the peak & Frequency in cm & $\begin{array}{l}\text { Assignment of } \\
\text { the peak }\end{array}$ \\
\hline 3942.46 & O-H stretching & 3343.20 & O-H stretching \\
\hline 3822.69 & O-H stretching & 3179.59 & O-H stretching \\
\hline 3717.77 & O-H stretching & 2923.45 & O-H stretching \\
\hline 3604.20 & O-H stretching & 2163.71 & C=C stretching \\
\hline 3498.57 & O-H stretching & 1637.31 & $\begin{array}{l}\text { C-H Finger print } \\
\text { region }\end{array}$ \\
\hline 3390.71 & O-H stretching & 1515.58 & C-C Stretching \\
\hline 2859.02 & C-H Stretching & 1439.41 & C=H Bending \\
\hline
\end{tabular}




\section{Results}

- The above analysis discuss that the spectrum of clove oil. The wave frequency $3942.46 \mathrm{~cm}^{-1}$ is obtained at the transmittance range of $78.83 \%$.

- Similarly the spectrum of Aspergillus fungi in the presence of clove oil, the wave frequency $3920.12 \mathrm{~cm}^{-1}$ is obtained at transmittance range of $36.23 \%$.

- FTIR,UV Studies of clove oil shows the vibrational assignments of Eugenol ,and its fluoresent property .The application of clove oil with the property of aroma is also experimentally proved by the method of vibrational analysis

- The analysis spectrum of Aspergillus fungi in the presence of clove oil in bread is observed. The result shows that growthof aspergillus fungai is reduced because of the application of clove oil.

\section{Acknowledgement: The Authors are thankful to SAIF- IIT,CHENNAI,INDIA}

\section{References}

[1] K. Singh, M. S. Barwa, and P. Tyagi, "Synthesis, characterization and biological studies of Co(II), Ni(II), Cu(II) and Zn(II) complexes with bidentate Schiff bases derived by heterocyclicketone," European Journal of Medicinal Chemistry, vol. 41, no.1, pp. $147-153,2006$.

[2] J. A. Obaleye, J. F. Adediji, and M. A. Adebayo, "Synthesis and biological activities on metal complexes of 2,5-diamino-1,3,4thiadiazole derived from semicarbazide hydrochloride,"Molecules, vol. 16, no. 7, pp. 5861-5874, 2011.

[3] X. Tai, X. Yin, Q. Chen, and M. Tan, "Synthesis of some transition metal complexes of a novel Schiff base ligand derived from 2,2 -bis(p-methoxyphenylamine) and salicylicaldehyde," Molecules, vol. 8, no. 5, pp. 439-443, 2003.

[4] A. A. H. Kadhum, A. B. Mohamad, A. A. Al-Amiery, and M.S. Takriff, "Antimicrobial and antioxidant activities of new metal complexes derived from 3-aminocoumarin," Molecules, vol. 16, no. 8, pp. 6969-6984, 2011.

[5] A. Corona-Bustamante, J.M. Viveros-Paredes, A. Flores-Parra et al., "Antioxidant activity of butyl- and phenylstannoxanes derived from2-, 3- and 4-pyridinecarboxylic acids," Molecules, vol. 15, no. 8, pp. 5445-5459, 2010.

[6.] Altantar, N, Soner, O., Tanker, M., et al., Antimicrobial activity of some volatile oils Journals of Turkish Microbiological society.

[7.] Janssen, A.M, Sheffer, J, J.C, Baertheim Suendsen.A., Anti microbial activity of essential oils; A 1976 Literature review: Aspects of the test methods, Planter Med., 53,395-398 (1987).

[8]. Kurita, N,m Miyali, M., Kurane, R., Takhav Y., Antifungal activity of components of essential oils. Agri. Bio. Chem., 45, $945-952$ (1981). 